\title{
HYALURONIDASE SPREAD AND THE EFFECT OF SALICYLATES IN RHEUMATIC FEVER
}

BY

\author{
DONALD H. ATLAS, PETER GABERMAN, and HERMAN EISENBERG \\ From the Departments of Medicine, The Chicago Medical School, \\ the Cook County Hospital, and the Mount Sinai Hospital of \\ Chicago, Illinois, U.S.A.
}

Since the pioneer work of Duran-Reynals in 1928 (Duran-Reynals, 1942), there has been a considerable investigation of spreading factors (Haas, 1946; Meyer, 1947) and their antagonists (Mayer and Kull, 1947; Wattenberg and Glick, 1949; Jones, 1950), concerning especially the mucolytic polysaccharide " hyaluronidase" (Hobby and others, 1941). Guerra (1946a, b) determined resistance to hyaluronidase by calculating the area of spread of intradermal weals of hyaluronidase dye combinations. Guerra and Robles Gil (1946) claimed that active and healed rheumatic fever patients have abnormal and rapid spreading reactions to testicular hyaluronidase. They suggested, therefore, that in rheumatic fever the mesenchymal tissues are especially involved, and that the rapid spread of inflammatory agents is evidence of removal of a protective ground-substance barrier. They also reported that therapeutic doses of salicylates inhibited these enzymatic spreading reactions and they concluded that the salutary effect of salicylates is mediated by this inhibition.

Although Dorfman and others (1947) corroborated the inhibitory effects of salicylates on hyaluronidase by animal experiments in vitro and in vivo, the in vitro inactivation of the hyaluronidase did not occur unless the concentrations of salicylates were greater than the usual therapeutic level and within the range in which salicylates denature biologically active proteins.

Harris and Friedman (1949), using haemoglobin as an indicator, were unable to demonstrate an increased susceptibility to streptococcus hyaluronidase in the tissues of rheumatic subjects. Ragan (1948), using similar techniques, was also unable to confirm Guerra's results.

Recent interest in the relationship of the hyaluronic acid-hyaluronidase system to rheumatic fever has been stimulated by four observations (Waksman, 1949):

(1) hyaluronic acid is an important constituent of connective tissue, the site of the rheumatic process;

(2) haemolytic streptococci produce hyaluronic acid and hyaluronidase;

(3) hyaluronidase allegedly causes in the skin of patients with rheumatic fever spreading reactions which are inhibited by salicylates;

(4) hyaluronic acid increases the sedimentation rate in vivo (in the rat) and in vitro, while hyaluronidase decreases the sedimentation rate in vitro even in the blood of rheumatic fever patients. 


\section{HYALURONIDASE AND SALICYLATES IN RHEUMATIC FEVER 419}

Since the completion of our work, Shuman (1950) has studied the problem of inhibition of hyaluronidase, using fluorescein as an indicator dye, and has reported normal spreading reactions in eight untreated acute rheumatic fever subjects, in two patients with disseminated lupus erythematosus, and in four patients with rheumatic conditions other than rheumatic fever. He showed that in patients treated with salicylates, hyaluronidase was inhibited and the disappearance of the dye delayed. He also reported that intravenous heparin appeared to inhibit the action of hyaluronidase and that anti-histaminic drugs had no effect.

Our purposes in this study were:

(1) to determine whether subjects with acute rheumatic fever or other febrile conditions have an altered resistance to the spread of hyaluronidase as compared to normal subjects,

(2) to observe the effects of salicylates on the spread of hyaluronidase weals in patients with acute rheumatic fever and in normal subjects.

\section{Methods}

One ml. of a solution of a 1 per cent. Evans blue (T1824) in saline was added to 150 turbidity reducing units of hyaluronidase.* Intradermal weals were made in the volar surface of the right forearm by injecting exactly $0.1 \mathrm{ml}$. of the hyaluronidase/Evans blue solution, using tuberculin syringes, the plungers and tips of which were treated with bisilicon petcock grease to prevent leakage and facilitate the injection of accurate amounts. A $\frac{1}{4}$-in. 27-gauge intradermal needle was used. As controls, weals were produced on the corresponding site of the left forearm, using $0.1 \mathrm{ml}$. of the 1 per cent. sterile Evans blue solution in saline. Since it was found that the maximum spread took place after $24 \mathrm{hrs}$, all measurements were made at this time interval. The area of spread was calculated as suggested by Harris and Friedman (1949). The longest axis of the spread area and its perpendicular were measured at the end of $24 \mathrm{hrs}$. Since the area of an ellipse is computed by the formula:

Area $=\frac{\pi D d}{4}$ (where $D$ is the long axis, and $d$ the perpendicular), the hyaluronidase area can be obtained by multiplying the product of the two axes by $0.785 \mathrm{~mm}$. The area of spread is the hyaluronidase area minus the control area in square millimetres.

To control the joint pain in the rheumatic subjects prior to administration of salicylates, codeine was necessary on occasion. The level of salicylates in the blood was determined during the 24-hr period of spread (Brodie and others, 1944).

\section{Material}

The patients for this investigation, selected from the Children's Hospital and the adult wards of the Cook County Hospital, were Negro and white, and ranged from 5 to 43 years of age.

Group I: Sixteen subjects in various stages of acute rheumatic fever and its chronic sequelae. No salicylates or anti-rheumatic drugs were administered before or during the course of the test (Table I).

Group II: Eight subjects with acute rheumatic fever. The skin tests were performed before and repeated during the administration of salicylates. The level of blood salicylate was determined within the 24-hr test period (Brodie and others, 1944). Only typical cases of theumatic fever were selected. Borderline cases lacking the usual criteria were rejected from the study (Table II).

Group III: 28 adult patients with a wide variety of conditions, including cases of

\footnotetext{
" Schering Corporation "Hydase".
} 
acute rheumatoid arthritis, acute gastro-enteritis, pneumonia, active pulmonary tuberculosis, acute pelvic inflammatory disease, acute upper respiratory tract infections, and several of the collagen diseases. None of the patients in this group received salicylates before or during the test (Table III).

Group IV: Fourteen normal young adult males. The test was carried out before and repeated during the administration of therapeutic doses of salicylates. Salicylate blood levels were also obtained during the test period. To establish maximum concentration of salicylates in the tissues, the drug was given for $48 \mathrm{hrs}$ before the test period as well as during the period of reaction (Table IV). Typical spreading reactions of normal young adults before the administration of salicylates are shown in Figs 1 and 2.

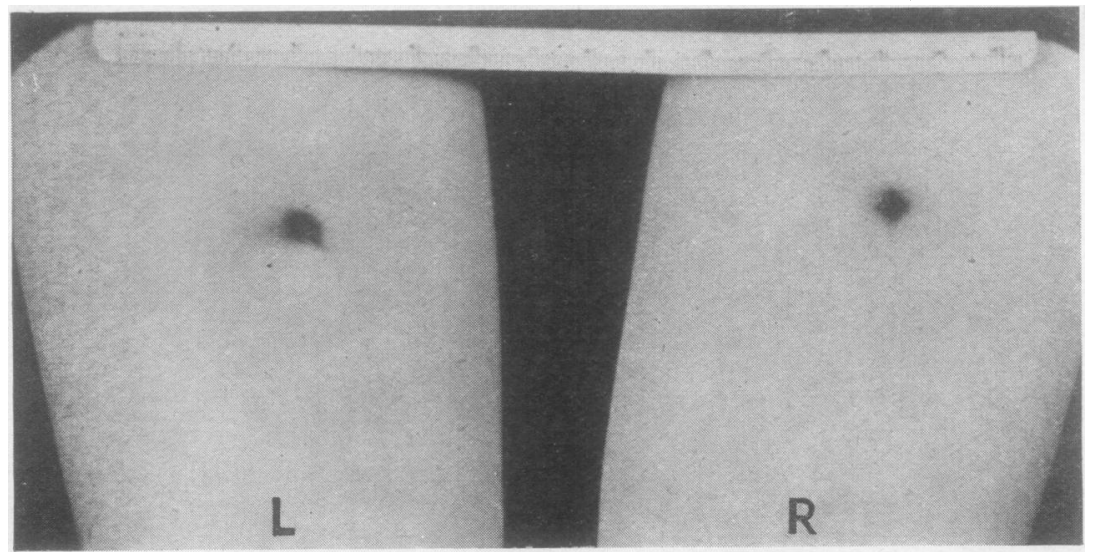

Fig. 1.-Normal young adult (not on salicylates) immediately after intradermal injection $0.1 \mathrm{ml}$. hyaluronidase/Evans blue solution (right forearm), $0.1 \mathrm{ml}$. 1 per cent. Evans blue solution in saline (left forearm).

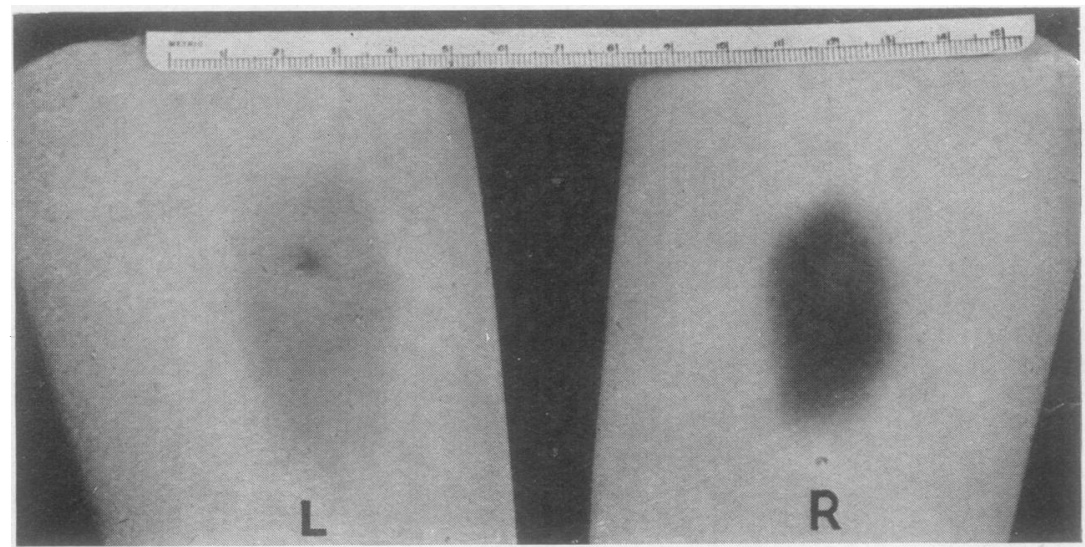

FIG. 2.-Same, $24 \mathrm{hrs}$ later, showing increased diffuision of dye in larger area of spread in right forearm showing hyaluronidase effect.

\section{Results}

Our results (see Tables) are in disagreement with all those of Guerra, and corroborate the observations of Harris and Friedman (1949), Ragan (1948), and Shuman (1950), in that we were unable to discern in rheumatic fever subjects any unusual 
HYALURONIDASE AND SALICYLATES IN RHEUMATIC FEVER 421

TABLE I

AREA OF SPREAD IN UNTREATED SUBJECTS WITH ACUTE RHEUMATIC FEVER

\begin{tabular}{|c|c|c|c|c|c|c|c|c|c|}
\hline \multirow{3}{*}{$\begin{array}{l}\text { Case } \\
\text { No. } \\
1\end{array}$} & \multirow{3}{*}{ Age } & \multirow{3}{*}{$\frac{\text { Race }}{\mathrm{C}}$} & \multirow{3}{*}{ Sex } & \multirow{2}{*}{$\begin{array}{l}\text { Manifestations of } \\
\text { Rheumatic Fever }\end{array}$} & \multicolumn{2}{|c|}{ Hyaluronidase } & \multicolumn{2}{|c|}{ Control } & \multirow{2}{*}{$\begin{array}{l}\text { Area of } \\
\text { Spread }\end{array}$} \\
\hline & & & & & $D$ & $d$ & $D$ & $d$ & \\
\hline & & & & $\begin{array}{l}\text { Acute migratory arthritis, febrile; } \\
\text { mitral stenosis; erythrocyte sedimen- } \\
\text { tation rate }-37 \mathrm{~mm} . / 1 \mathrm{hr}\end{array}$ & $4 \cdot 0$ & $2 \cdot 0$ & $2 \cdot 2$ & $1 \cdot 8$ & $3 \cdot 14$ \\
\hline 2 & 13 & C & $\mathrm{F}$ & $\begin{array}{l}\text { Acute febrile recurrent rheumatic } \\
\text { carditis with mild congestive failure }\end{array}$ & $5 \cdot 0$ & $3 \cdot 8$ & $4 \cdot 0$ & $2 \cdot 5$ & $7 \cdot 07$ \\
\hline 3 & 20 & C & $\mathbf{F}$ & $\begin{array}{l}\text { Subsiding rheumatic carditis and } \\
\text { arthritis; mitral configuration heart; } \\
\text { murmurs of mitral stenosis }\end{array}$ & $8 \cdot 0$ & $3 \cdot 0$ & $4 \cdot 5$ & $2 \cdot 0$ & $11 \cdot 7$ \\
\hline 4 & 22 & $\mathrm{C}$ & $\mathbf{F}$ & $\begin{array}{l}\text { Acute febrile rheumatic fever with } \\
\text { migratory arthritis }\end{array}$ & $6 \cdot 8$ & $2 \cdot 3$ & $2 \cdot 8$ & $2 \cdot 1$ & $7 \cdot 62$ \\
\hline 5 & 13 & $\mathbf{W}$ & $\mathbf{M}$ & $\begin{array}{l}\text { Acute rheumatic fever with migra- } \\
\text { tory arthritis; systolic murmur of } \\
\text { apex }\end{array}$ & $6 \cdot 0$ & $4 \cdot 0$ & $3 \cdot 5$ & $2 \cdot 3$ & $12 \cdot 5$ \\
\hline 6 & 43 & W & $\mathbf{M}$ & $\begin{array}{l}\text { Subsiding acute rheumatic fever; } \\
\text { migratory arthritis; mitral stenosis }\end{array}$ & $5 \cdot 5$ & $4 \cdot 5$ & $3 \cdot 5$ & $2 \cdot 7$ & $12 \cdot 04$ \\
\hline 7 & 11 & W & $\mathbf{M}$ & $\begin{array}{l}\text { Acute rheumatic carditis with migra- } \\
\text { tory polyarthritis; mitral stenosis }\end{array}$ & $5 \cdot 0$ & $3 \cdot 1$ & $2 \cdot 6$ & $1 \cdot 7$ & $8 \cdot 72$ \\
\hline 8 & 8 & C & $\mathbf{F}$ & $\begin{array}{l}\text { Haemolytic streptococcal pharyn- } \\
\text { gitis; febrile; migratory arthritis; } \\
\text { mitral insufficiency }\end{array}$ & $4 \cdot 3$ & $2 \cdot 2$ & $2 \cdot 7$ & $1 \cdot 8$ & $3 \cdot 82$ \\
\hline 9 & 11 & C & $\mathbf{M}$ & $\begin{array}{l}\text { Acute rheumatic migratory poly- } \\
\text { arthritis with carditis; mitral insuff- } \\
\text { ciency; febrile }\end{array}$ & $6 \cdot 2$ & $3 \cdot 0$ & $4 \cdot 5$ & $2 \cdot 2$ & $6 \cdot 83$ \\
\hline 10 & 11 & C & $\mathrm{F}$ & $\begin{array}{l}\text { Haemolytic streptococcal pharyn- } \\
\text { gitis; febrile; migratory polyarthritis; } \\
\text { carditis; prolonged P-R interval }\end{array}$ & $4 \cdot 0$ & $2 \cdot 6$ & $3 \cdot 4$ & $2 \cdot 6$ & $1 \cdot 22$ \\
\hline 11 & 11 & $\mathbf{C}$ & $\mathbf{F}$ & $\begin{array}{l}\text { Recurrent rheumatic polyarthritis, } \\
\text { acute; febrile; sedimentation rate- } \\
60 \mathrm{~mm} . / 1 \mathrm{hr} \text {; mitral insufficiency }\end{array}$ & $7 \cdot 0$ & $3 \cdot 0$ & $3 \cdot 0$ & $2 \cdot 0$ & $11 \cdot 5$ \\
\hline 12 & 14 & W & $\mathbf{F}$ & $\begin{array}{l}\text { Migratory polyarthritis; febrile- } \\
2 \text { months, subsiding: no evidence } \\
\text { cardiac involvement }\end{array}$ & $4 \cdot 0$ & $3 \cdot 0$ & $4 \cdot 0$ & $2 \cdot 5$ & $1 \cdot 57$ \\
\hline 13 & 7 & C & $\mathbf{M}$ & $\begin{array}{l}\text { Severe rheumatic pancarditis with } \\
\text { pericardial effusion; acutely ill }\end{array}$ & $4 \cdot 5$ & $3 \cdot 7$ & $2 \cdot 5$ & $2 \cdot 0$ & $9 \cdot 2$ \\
\hline 14 & 8 & W & $\mathrm{F}$ & $\begin{array}{l}\text { Admitted for arrhythmia; auricular } \\
\text { fibrillation; aortic and mitral valve } \\
\text { involvement ; febrile ; subsiding } \\
\text { carditis }\end{array}$ & $4 \cdot 5$ & $3 \cdot 3$ & $3 \cdot 2$ & $2 \cdot 2$ & $6 \cdot 2$ \\
\hline 15 & 11 & C & $\mathbf{M}$ & $\begin{array}{l}\text { Left unilateral hemi-chorea- } \\
3 \text { weeks; no previous history or } \\
\text { findings of rheumatic fever; afebrile }\end{array}$ & $4 \cdot 5$ & $2 \cdot 2$ & $1 \cdot 2$ & $1 \cdot 0$ & $6 \cdot 83$ \\
\hline 16 & 5 & C & $\mathrm{F}$ & $\begin{array}{l}\text { Acute febrile migratory polyarthritis; } \\
\text { sedimentation rate }-31 \mathrm{~mm} . / 1 \mathrm{hr} \text {. }\end{array}$ & $5 \cdot 0$ & $2 \cdot 0$ & $3 \cdot 0$ & $2 \cdot 0$ & $3 \cdot 14$ \\
\hline
\end{tabular}

or excessive spreading reactions with hyaluronidase. The administration of salicylates to rheumatic fever subjects produced no greater reduction of area of spread than was observed in the normal group. The area of spread with hyaluronidase in normal subjects was found to extend over a wide range from $7 \cdot 3$ 
TABLE II

AREA OF SPREAD IN SUBJECTS WITH ACUTE RHEUMATIC FEVER BEFORE AND DURING ADMINISTRATION OF SODIUM SALICYLATE

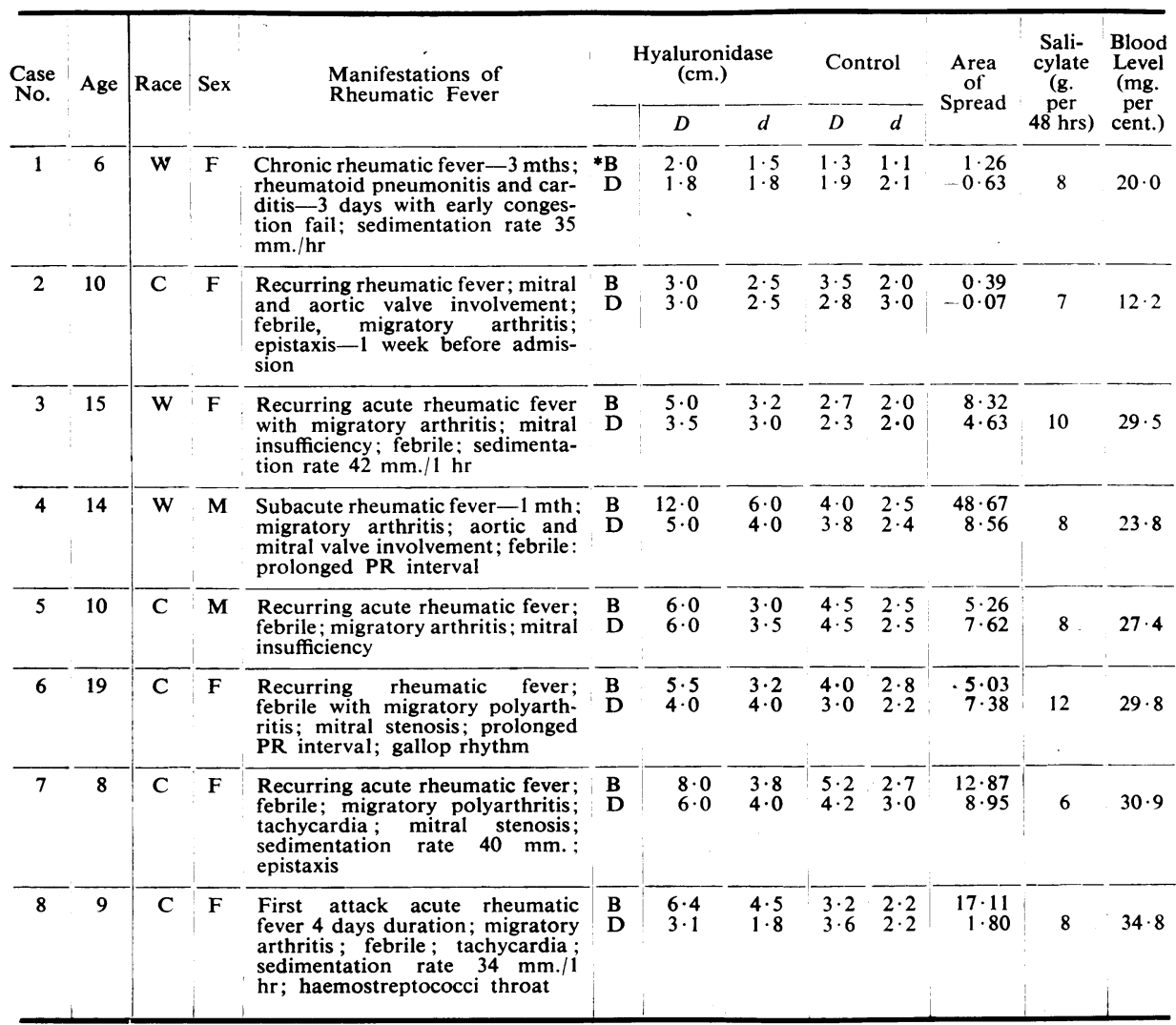

*B - before administration of salicylate.

D-during administration of salicylate.

to 53.4 sq. mm., 16 being the arithmetical average for fourteen normal subjects. With such a wide span of values in the normal group, the discernment of statistically significant areas of spread in pathological conditions is obviously inconclusive. In general, spreading reactions in the acute rheumatic fever group were not significantly different from those in the normal group. The controlled administration of therapeutic doses of salicylates to eight subjects with acute rheumatic fever (Group II) reduced the area of spread significantly in six cases, whereas in two cases the area of spread was slightly larger during salicylate administration. Spreading reactions in subjects with inflammatory conditions and collagen diseases (Group III)* were also within the normal range. This is especially noteworthy in the several patients with collagen diseases.

* Case 14 (Group III), with active hyperthyroidism, had an excessively large area of spread which may be attributed to the increased vascularity of the tissues rather than decreased resistance to hyaluronidase. 
TABLE III

AREA OF SPREAD IN SUBJECTS WITH INFLAMMATORY

CONDITIONS AND COLLAGEN DISEASES

\begin{tabular}{|c|c|c|c|c|c|c|c|c|c|c|}
\hline \multirow{2}{*}{$\begin{array}{l}\text { Case } \\
\text { No. }\end{array}$} & \multirow{2}{*}{ Age } & \multirow{2}{*}{ Race } & \multirow{2}{*}{ Sex } & \multirow{2}{*}{ Diagnosis } & \multirow{2}{*}{$\begin{array}{l}\text { Tempera- } \\
\text { ture }\end{array}$} & \multicolumn{2}{|c|}{ Hyaluronidase } & \multicolumn{2}{|c|}{ Control } & \multirow{2}{*}{$\begin{array}{c}\text { Area } \\
\text { of } \\
\text { Spread }\end{array}$} \\
\hline & & & & & & $D$ & $d$ & $D$ & $d$ & \\
\hline 1 & 20 & $\mathrm{C}$ & $\mathbf{F}$ & Acute nonspecific gastro-enteritis & $102^{\circ} \mathrm{F}$ & $4 \cdot 5$ & $3 \cdot 3$ & $3 \cdot 5$ & $2 \cdot 3$ & $5 \cdot 34$ \\
\hline 2 & 50 & W & $\mathbf{F}$ & $\begin{array}{l}\text { Lobar pneumonia, acute, right and left } \\
\text { lungs }\end{array}$ & $101^{\circ} \mathrm{F}$ & $5 \cdot 5$ & $3 \cdot 5$ & $4 \cdot 0$ & $2 \cdot 8$ & $6 \cdot 36$ \\
\hline 3 & 26 & $\mathbf{C}$ & $\mathbf{F}$ & Pulmonary tuberculosis, F.A.B. & $102^{\circ} \mathrm{F}$ & $6 \cdot 0$ & $3 \cdot 0$ & $4 \cdot 0$ & $2 \cdot 5$ & $6 \cdot 28$ \\
\hline 4 & 32 & $\mathbf{C}$ & $\mathbf{F}$ & Pulmonary tuberculosis, unilateral, F.A. & $99 \cdot 6^{\circ} \mathrm{F}$ & $6 \cdot 0$ & $2 \cdot 2$ & $3 \cdot 0$ & $2 \cdot 2$ & $5 \cdot 18$ \\
\hline 5 & 20 & $\mathbf{C}$ & $\mathbf{F}$ & $\begin{array}{l}\text { Pelvic inflammatory disease, subacute; } \\
\text { with pregnancy }-3 \text { months }\end{array}$ & Afebrile & $4 \cdot 2$ & $4 \cdot 0$ & $2 \cdot 7$ & $2 \cdot 2$ & $8 \cdot 56$ \\
\hline 6 & 56 & $\mathrm{C}$ & F. & $\begin{array}{l}\text { Fever of undetermined origin; suspected } \\
\text { periarteritis nodosa }\end{array}$ & $100 \cdot 2^{\circ} \mathrm{F}$ & $8 \cdot 0$ & $4 \cdot 5$ & $5 \cdot 0$ & $2 \cdot 2$ & $19 \cdot 62$ \\
\hline 7 & 34 & $\mathbf{C}$ & $\mathbf{F}$ & $\begin{array}{l}\text { Tuberculous bronchopneumonia; RUL } \\
\text { acute }\end{array}$ & $106^{\circ} \mathrm{F}$ & $4 \cdot 0$ & $2 \cdot 6$ & $3 \cdot 2$ & $2 \cdot 3$ & $2 \cdot 35$ \\
\hline 8 & 23 & $\mathbf{w}$ & $\mathbf{F}$ & $\begin{array}{l}\text { Chronic amoebiasis; tuberculous pleural } \\
\text { effusion }\end{array}$ & Afebrile & $5 \cdot 0$ & $3 \cdot 0$ & $3 \cdot 0$ & $2 \cdot 0$ & $7 \cdot 07$ \\
\hline 9 & 24 & $\mathbf{C}$ & $\mathbf{F}$ & Acute hepatitis & $101 \cdot 6^{\circ} \mathrm{F}$ & $2 \cdot 0$ & $2 \cdot 0$ & $3 \cdot 8$ & $2 \cdot 0$ & $-2 \cdot 83$ \\
\hline 10 & 45 & $\mathbf{W}$ & $\mathbf{F}$ & $\begin{array}{l}\text { Chronic alcoholism; Laennec's cirrhosis } \\
\text { with jaundice }\end{array}$ & Afebrile & $4 \cdot 5$ & $2 \cdot 5$ & $5 \cdot 0$ & $2 \cdot 5$ & -0.94 \\
\hline 11 & 26 & $\mathbf{W}$ & $\mathbf{F}$ & Obstructive jaundice & Afebrile & $4 \cdot 0$ & $2 \cdot 5$ & $2 \cdot 2$ & $1 \cdot 8$ & $4 \cdot 74$ \\
\hline 12 & 42 & C & $\mathbf{F}$ & Acute hepatitis with jaundice & Afebrile & $4 \cdot 7$ & $3 \cdot 5$ & $4 \cdot 0$ & $2 \cdot 7$ & $4 \cdot 47$ \\
\hline 13 & 22 & $\mathbf{C}$ & $\mathbf{F}$ & Acute upper respiratory tract infection & $102^{\circ} \mathrm{F}$ & $10 \cdot 9$ & $3 \cdot 8$ & $4 \cdot 4$ & $2 \cdot 6$ & $23 \cdot 55$ \\
\hline 14 & 36 & $\mathbf{C}$ & $\mathbf{F}$ & $\begin{array}{l}\text { Thyrotoxicosis, severe; diffuse enlarge- } \\
\text { ment of thyroid }\end{array}$ & Afebrile & $9 \cdot 5$ & $4 \cdot 8$ & $5 \cdot 7$ & $2 \cdot 9$ & $32 \cdot 65$ \\
\hline 15 & 57 & $\mathbf{w}$ & $\mathbf{F}$ & Obstructive jaundice & Afebrile & $5 \cdot 8$ & $3 \cdot 5$ & $2 \cdot 7$ & $2 \cdot 6$ & $10 \cdot 44$ \\
\hline 16 & 22 & $\mathbf{C}$ & $\mathbf{F}$ & Tuberculosis, pulmonary F.A. bilateral & $100 \cdot 8^{\circ} \mathrm{F}$ & $7 \cdot 0$ & $2 \cdot 5$ & $4 \cdot 0$ & 1.8 & 8.09 \\
\hline 17 & 13 & $\mathbf{C}$ & $\mathbf{F}$ & Acute tracheal bronchitis & $102^{\circ} \mathrm{F}$ & $3 \cdot 0$ & $2 \cdot 0$ & $2 \cdot 2$ & $1 \cdot 5$ & $2 \cdot 12$ \\
\hline 18 & 14 & $\mathbf{C}$ & $\mathbf{F}$ & Infectious hepatitis with jaundice & Afebrile & $5 \cdot 0$ & $4 \cdot 0$ & $3 \cdot 8$ & $2 \cdot 2$ & 0.48 \\
\hline 19 & 20 & Mex. & $\mathbf{F}$ & Alcoholic cirrhosis with jaundice & 一 & $5 \cdot 0$ & $3 \cdot 8$ & $4 \cdot 0$ & $3 \cdot 0$ & $5 \cdot 6$ \\
\hline 20 & 21 & $\mathbf{w}$ & $\mathbf{F}$ & $\begin{array}{l}\text { Chronic glomerulonephritis and severe } \\
\text { azotaemia }\end{array}$ & - & $4 \cdot 7$ & $2 \cdot 6$ & $3 \cdot 5$ & $2 \cdot 5$ & $1 \cdot 72$ \\
\hline 21 & 19 & $\mathbf{W}$ & $\mathbf{F}$ & $\begin{array}{l}\text { Chronic atrophic long-standing rheuma- } \\
\text { toid arthritis }\end{array}$ & Afebrile & $3 \cdot 5$ & $2 \cdot 0$ & $2 \cdot 2$ & $1 \cdot 2$ & $3 \cdot 43$ \\
\hline 22 & 19 & $\mathbf{C}$ & $\mathbf{F}$ & $\begin{array}{l}\text { Acute monoarticular inflammatory arth- } \\
\text { ritis, cause undetermined }\end{array}$ & - & $5 \cdot 0$ & $4 \cdot 5$ & $3 \cdot 0$ & $2 \cdot 0$ & $12 \cdot 95$ \\
\hline 23 & 39 & $\mathbf{C}$ & $\mathbf{F}$ & Acute infectious arthritis & $101^{\circ} \mathrm{F}$ & $4 \cdot 5$ & $2 \cdot 2$ & $3 \cdot 0$ & $2 \cdot 0$ & 3.06 \\
\hline 24 & 26. & $\mathbf{w}$ & $\mathbf{F}$ & Acute infectious arthritis & $100^{\circ} \mathrm{F}$ & $6 \cdot 8$ & $4 \cdot 7$ & $3 \cdot 7$ & $2 \cdot 5$ & $17 \cdot 83$ \\
\hline 25 & 20 & $\mathbf{C}$ & $\mathbf{F}$ & $\begin{array}{l}\text { Acute lupus erythematosus disseminatus. } \\
\text { LE cells in marrow }\end{array}$ & $103^{\circ} \mathrm{F}$ & $9 \cdot 0$ & $4 \cdot 5$ & $5 \cdot 5$ & $2 \cdot 5$ & $21 \cdot 0$ \\
\hline 26 & 26 & $\mathbf{W}$ & $\mathbf{F}$ & $\begin{array}{l}\text { Subacute lupus erythematosus dis- } \\
\text { seminatus. LE cells in marrow }\end{array}$ & Afebrile & $4 \cdot 5$ & $3 \cdot 8$ & $3 \cdot 0$ & $2 \cdot 3$ & $8 \cdot 0$ \\
\hline 27 & 41 & $\mathbf{w}$ & $\mathbf{F}$ & $\begin{array}{l}\text { Acute lupus erythematosus disseminatus. } \\
\text { LE cells in marrow }\end{array}$ & - & $7 \cdot 0$ & $3 \cdot 5$ & $4 \cdot 5$ & $2 \cdot 5$ & $10 \cdot 36$ \\
\hline 28 & 19 & $\mathbf{w}$ & $\mathbf{F}$ & $\begin{array}{l}\text { Lupus erythematosus disseminatus, acute } \\
\text {-terminal; LE cells in marrow }\end{array}$ & - & $3 \cdot 0$ & $1 \cdot 5$ & $2 \cdot 0$ & $1 \cdot 0$ & 1.96 \\
\hline
\end{tabular}

In ten of the fourteen normal controls (Group IV), administration of salicylates significantly reduced the area of the spreading reaction. In four of this group, the area of spread after the administration of salicylates was the same or even larger, 
TABLE IV

AREA OF SPREAD BEFORE AND DURING ADMINISTRATION OF SODIUM SALICYLATE IN NORMAL CONTROLS

\begin{tabular}{|c|c|c|c|c|c|c|c|c|}
\hline \multirow{2}{*}{$\begin{array}{l}\text { Case } \\
\text { No. }\end{array}$} & \multirow{2}{*}{$\begin{array}{l}\text { Adminis- } \\
\text { tration of } \\
\text { Salicylate }\end{array}$} & \multicolumn{2}{|c|}{ Hyaluronidase } & \multicolumn{2}{|c|}{ Control } & \multirow{2}{*}{$\begin{array}{l}\text { Area of } \\
\text { Spread }\end{array}$} & \multirow{2}{*}{$\begin{array}{l}\text { Salicylate } \\
\text { (g. } / 72 \text { hrs) }\end{array}$} & \multirow{2}{*}{$\begin{array}{l}\text { Blood Level } \\
\text { (mg. per cent.) }\end{array}$} \\
\hline & & $D$ & $d$ & $D$ & $d$ & & & \\
\hline 1 & $\begin{array}{r}* \mathrm{~B} \\
\mathrm{D}\end{array}$ & $\begin{array}{l}15 \cdot 0 \\
13 \cdot 0\end{array}$ & $\begin{array}{l}6 \cdot 0 \\
5 \cdot 8\end{array}$ & $\begin{array}{l}7 \cdot 0 \\
8 \cdot 0\end{array}$ & $\begin{array}{l}3 \cdot 2 \\
4 \cdot 0\end{array}$ & $\begin{array}{l}53 \cdot 4 \\
34 \cdot 1\end{array}$ & $9 \cdot 3$ & $22 \cdot 8$ \\
\hline 2 & $\begin{array}{l}\text { B } \\
\text { D }\end{array}$ & $\begin{array}{l}7 \cdot 0 \\
4 \cdot 0\end{array}$ & $\begin{array}{l}4 \cdot 3 \\
3 \cdot 5\end{array}$ & $\begin{array}{l}3 \cdot 5 \\
3 \cdot 0\end{array}$ & $\begin{array}{l}3 \cdot 0 \\
2 \cdot 0\end{array}$ & $\begin{array}{r}15 \cdot 4 \\
6 \cdot 3\end{array}$ & $22 \cdot 0$ & $38 \cdot 0$ \\
\hline 3 & $\begin{array}{l}\text { B } \\
\text { D }\end{array}$ & $\begin{array}{l}7 \cdot 0 \\
4 \cdot 5\end{array}$ & $\begin{array}{l}3 \cdot 0 \\
2 \cdot 3\end{array}$ & $\begin{array}{l}4 \cdot 5 \\
3 \cdot 5\end{array}$ & $\begin{array}{l}2 \cdot 5 \\
1 \cdot 8\end{array}$ & $\begin{array}{l}7 \cdot 6 \\
3 \cdot 2\end{array}$ & $19 \cdot 3$ & $22 \cdot 5$ \\
\hline 4 & $\begin{array}{l}\mathrm{B} \\
\mathrm{D}\end{array}$ & $\begin{array}{l}6 \cdot 0 \\
5 \cdot 8\end{array}$ & $\begin{array}{l}5 \cdot 0 \\
4 \cdot 5\end{array}$ & $\begin{array}{l}4 \cdot 5 \\
4 \cdot 5\end{array}$ & $\begin{array}{l}2 \cdot 8 \\
3 \cdot 5\end{array}$ & $\begin{array}{r}13 \cdot 7 \\
8 \cdot 1\end{array}$ & $12 \cdot 0$ & $9 \cdot 0$ \\
\hline 5 & $\begin{array}{l}\text { B } \\
\text { D }\end{array}$ & $\begin{array}{l}7 \cdot 0 \\
6 \cdot 3\end{array}$ & $\begin{array}{l}5 \cdot 0 \\
3 \cdot 5\end{array}$ & $\begin{array}{l}4 \cdot 8 \\
4 \cdot 0\end{array}$ & $\begin{array}{l}3 \cdot 2 \\
2 \cdot 7\end{array}$ & $\begin{array}{r}15 \cdot 4 \\
8 \cdot 9\end{array}$ & $22 \cdot 0$ & $26 \cdot 7$ \\
\hline 6 & $\begin{array}{l}\mathrm{B} \\
\mathrm{D}\end{array}$ & $\begin{array}{l}6 \cdot 5 \\
6 \cdot 5\end{array}$ & $\begin{array}{l}5 \cdot 0 \\
4 \cdot 5\end{array}$ & $\begin{array}{l}4 \cdot 5 \\
5 \cdot 3\end{array}$ & $\begin{array}{l}3 \cdot 0 \\
4 \cdot 8\end{array}$ & $\begin{array}{r}14 \cdot 9 \\
3 \cdot 1\end{array}$ & $29 \cdot 0$ & $33 \cdot 0$ \\
\hline 7 & $\begin{array}{l}\text { B } \\
\text { D }\end{array}$ & $\begin{array}{l}9 \cdot 0 \\
7 \cdot 2\end{array}$ & $\begin{array}{l}5 \cdot 0 \\
4 \cdot 2\end{array}$ & $\begin{array}{l}4 \cdot 0 \\
4 \cdot 2\end{array}$ & $\begin{array}{l}2 \cdot 5 \\
3 \cdot 7\end{array}$ & $\begin{array}{l}27 \cdot 5 \\
11 \cdot 5\end{array}$ & $14 \cdot 6$ & $21 \cdot 0$ \\
\hline 8 & $\begin{array}{l}\mathrm{B} \\
\mathrm{D}\end{array}$ & $\begin{array}{l}5 \cdot 8 \\
6 \cdot 9\end{array}$ & $\begin{array}{l}4 \cdot 0 \\
3 \cdot 8\end{array}$ & $\begin{array}{l}4 \cdot 9 \\
4 \cdot 6\end{array}$ & $\begin{array}{l}3 \cdot 0 \\
3 \cdot 0\end{array}$ & $\begin{array}{l}6 \cdot 7 \\
9 \cdot 7\end{array}$ & $22 \cdot 6$ & $25 \cdot 8$ \\
\hline 9 & $\begin{array}{l}\text { B } \\
\text { D }\end{array}$ & $\begin{array}{l}9 \cdot 7 \\
7 \cdot 5\end{array}$ & $\begin{array}{l}4 \cdot 8 \\
5 \cdot 0\end{array}$ & $\begin{array}{l}6 \cdot 3 \\
4 \cdot 3\end{array}$ & $\begin{array}{l}4 \cdot 0 \\
3 \cdot 2\end{array}$ & $\begin{array}{l}16 \cdot 8 \\
18 \cdot 6\end{array}$ & $24 \cdot 0$ & $37 \cdot 6$ \\
\hline 10 & $\begin{array}{l}\text { B } \\
\text { D }\end{array}$ & $\begin{array}{l}7 \cdot 5 \\
5 \cdot 3\end{array}$ & $\begin{array}{l}4 \cdot 2 \\
3 \cdot 5\end{array}$ & $\begin{array}{l}3 \cdot 5 \\
3 \cdot 6\end{array}$ & $\begin{array}{l}3 \cdot 0 \\
2 \cdot 2\end{array}$ & $\begin{array}{r}16 \cdot 5 \\
8 \cdot 4\end{array}$ & $30 \cdot 0$ & $31 \cdot 1$ \\
\hline 11 & $\begin{array}{l}\text { B } \\
\text { D }\end{array}$ & $\begin{array}{l}6 \cdot 5 \\
6 \cdot 5\end{array}$ & $\begin{array}{l}4 \cdot 2 \\
4 \cdot 5\end{array}$ & $\begin{array}{l}4 \cdot 5 \\
5 \cdot 0\end{array}$ & $\begin{array}{l}4 \cdot 0 \\
2 \cdot 8\end{array}$ & $\begin{array}{r}7 \cdot 3 \\
12 \cdot 0\end{array}$ & $30 \cdot 0$ & $24 \cdot 4$ \\
\hline 12 & $\begin{array}{l}\text { B } \\
\text { D }\end{array}$ & $\begin{array}{l}7 \cdot 5 \\
4 \cdot 5\end{array}$ & $\begin{array}{l}5 \cdot 0 \\
3 \cdot 0\end{array}$ & $\begin{array}{l}5 \cdot 5 \\
3 \cdot 7\end{array}$ & $\begin{array}{l}3 \cdot 0 \\
2 \cdot 5\end{array}$ & $\begin{array}{r}16 \cdot 5 \\
3 \cdot 5\end{array}$ & $30 \cdot 0$ & $30 \cdot 1$ \\
\hline 13 & $\begin{array}{l}\text { B } \\
\text { D }\end{array}$ & $\begin{array}{l}7 \cdot 5 \\
7 \cdot 5\end{array}$ & $\begin{array}{l}3 \cdot 2 \\
4 \cdot 5\end{array}$ & $\begin{array}{l}4 \cdot 8 \\
4 \cdot 6\end{array}$ & $\begin{array}{l}3 \cdot 0 \\
2 \cdot 2\end{array}$ & $\begin{array}{l}7 \cdot 5 \\
8 \cdot 6\end{array}$ & $23 \cdot 3$ & $21 \cdot 2$ \\
\hline 14 & $\begin{array}{l}\text { B } \\
\text { D }\end{array}$ & $\begin{array}{l}8 \cdot 0 \\
7 \cdot 5\end{array}$ & $\begin{array}{l}3 \cdot 5 \\
4 \cdot 0\end{array}$ & $\begin{array}{l}4 \cdot 0 \\
4 \cdot 8\end{array}$ & $\begin{array}{l}4 \cdot 0 \\
3 \cdot 0\end{array}$ & $\begin{array}{r}9 \cdot 4 \\
12 \cdot 3\end{array}$ & $20 \cdot 0$ & $23 \cdot 5$ \\
\hline
\end{tabular}

indicating that there is a considerable variation in anti-hyaluronidase factors in normal individuals. Assay of anti-hyaluronidase factors (Haas, 1946; Harris and others, 1950; Thompson and Moses, 1949) would have been highly valuable but was not obtainable in this project.

\section{Comment}

The only explanation we have to offer for the discrepancy between Guerra's results and our own is that he may have been dealing with an impure enzyme 
containing other antigenic substances which produced the massive reactions he described. Also, Guerra does not report the controlled use of his material in normal individuals or in subjects with other diseases. Although our work was done with testicular hyaluronidase, Harris and also Ragan were unable to demonstrate increased susceptibility of rheumatic subjects to streptococcus hyaluronidase.

Our results confirm the observations of Dorfman and others (1947), and of Harris and others (1950) that salicylates inhibit spreading enzymes. That this inhibitory action of salicylates was not found in all subjects-normal and rheumatic -indicates that in some individuals other anti-hyaluronidase factors may be operative in varying degrees. Our results, however, do suggest that the beneficial effect of salicylates in rheumatic fever is mediated through the ground substance.

\section{Summary}

Intradermal injections of $0 \cdot 1 \mathrm{ml}$. hyaluronidase/Evans blue solution, and control injections of Evans blue in saline, were made in four types of subjects:

(a) various phases of acute and quiescent rheumatic fever (Groups I and II),

(b) various inflammatory conditions,

(c) various collagen diseases,

(Group III)

(d) normal controls (Group IV).

No significant alteration in resistance to the spread of hyaluronidase could be discerned in any of the conditions studied, as shown by the size of the resulting weals.

Spreading reactions were measured before and during the administration of therapeutic doses of salicylates to a group of patients with acute rheumatic fever (Group II), and to control subjects (Group IV). Spreading reactions were found to be inhibited by salicylates in most subjects, but the effect of salicylates on this inhibition is no greater in rheumatic fever subjects than in normal subjects.

It is suggested that salicylates exert their therapeutic action directly on the ground substance by virtue of their inhibitory effect on spreading enzymes.

We wish to thank the Department of Pediatrics of the Cook County Hospital for permission to study some of their cases of rheumatic fever.

\section{REFERENCES}

Brodie, B. B., Udenfriend, S., and Coburn, A. F. (1944). J. Pharmacol., 80, 114.

Dorfman, A., Reimers, E. J., and Ott, M. L. (1947). Proc. Soc. Exp. Biol., N.Y., 64, 357.

Duran-Reynals, F. (1942). Bact. Rev., 6, 197.

Guerra, F. (1946a). Science, 103, 686.

- (1946b). J. Pharmacol., 87, 193.

—, and Robles Gil, J. (1946). Arch. Inst. cardiol. Méx., 16, 293.

Haas, E. (1946). J. biol. Chem., 163, 63.

Harris, T. N., and Friedman, S. (1949). Amer. J. Dis. Child., 77, 561.

- Harris, S., Dannenberg, A. M., and Hollander, J. L. (1950). Ann. intern. Med., $32,917$.

Hobby, G. L., Dawson, M. H., Meyer, K., and Chaffee, E. (1941). J. exp. Med., 73, 109.

Jones, E. S. (1950). Annals of the Rheumatic Diseases, 9, 137.

Mayer, R. L., and Kull, F. C. (1947). Proc. Soc. exp. Biol., N.Y., 66, 392.

Meyer, K. (1947). Physiol. Rev., 27, 335. 
Ragan, C. (1948). Conference on the Ground Substance of the Mesenchyme and Hyaluronidase, Dec. 3 and 4, 1948. (To be published in Ann. N.Y. Acad. Sci.)

Shuman, C. R. (1950). Amer. J. med. Sci., 220, 665.

Thompson, R. T., and Moses, F. E. (1949). Science, 110, 70.

Waksman, B. H. (1949). Medicine, Baltimore, 28, 143.

Wattenberg, L. W., and Glick, D. (1949). J. biol. Chem., 179, 1213.

\section{La diffusion de l'hyaluronidase et l'effet des salicylates} dans le rhumatisme articulaire aigu

\section{RÉSUMÉ}

Des injections intradermiques de $0.1 \mathrm{ml}$. d'hyaluronidase/bleu d'Evans et des injections de contrôle de bleu d'Evans dans l'eau physiologique furent effectuées chez des sujets appartenant à un des quatre groupes suivantes:

(a) rhumatisme articulaire aigu à la période d'état et d'abattement (Groupes I et II),

(b) affections inflammatoires diverses

(c) maladies collagènes diverses $\}$ (Groupe III),

(d) individus sains (Groupe IV).

En se basant sur les dimensions des taches produites on n'a remarqué aucune altération significative de la résistance à la diffusion de l'hyaluronidase dans les affections étudiées.

La diffusion fut mesurée avant et pendant l'administration de doses thérapeutiques de salicylates aux malades atteints de rhumatisme articulaire aigu (Groupe II) et aux témoins (Groupe IV). On a trouvé que les salicylates inhibaient la diffusion chez la plupart des sujets, mais que cette inhibition n'était pas plus accentuée chez les rhumatisants que chez les témoins.

On suggère que les salicylates exercent leur action thérapeutique directément sur la substance basique, en inhibant la diffusion de l'hyaluronidase.

\section{La difusión de la hialuronidasa y el efecto de los salicilatos en el reumatismo articular agudo}

\section{Sumario}

Inyecciones intradérmicas de $0,1 \mathrm{ml}$. de hialuronidasa/azul de Evans e inyecciones de control de azul de Evans en suero fisiológico fueron aplicadas a sujetos de cuatro grupos:

(a) reumatismo articular agudo en el periódo de estado y de remisión (Grupos I y II),

(b) varias afecciones inflamatorias $\}$

(c) varias enfermedades colágenas $\}$ (Grupo III),

(d) individuos sanos (Grupo IV).

A juzgar por las dimensiones de las manchas producidas no hubo alteración significante de la resistencia a la difusión de la hialuronidasa en las afecciones estudiadas.

El area de la difusión fué determinada antes y después de la administración de dosis terapéuticas de salicilatos a enfermos con reumatismo articular agudo (Grupo II) y a los controles (Grupo IV). Se averiguó que los salicilatos inhibían la difusión en la mayoría de los sujetos, pero que esta inhibición no fué más acentuada en los enfermos con reumatismo que en los sanos.

Se sugiere que los salicilatos ejercen su acción terapéutica directamente sobre la substancia basal por inhibición de la difusión de la hialuronidasa. 